\title{
Effect of Fock exchange on the electronic structure and magnetic coupling in $\mathrm{NiO}$
}

\author{
Ibério de P. R. Moreira, ${ }^{1}$ Francesc Illas, ${ }^{1}$ and Richard L. Martin ${ }^{2}$ \\ ${ }^{1}$ Departament de Química Física i Centre de Recerca en Química Teòrica, Universitat de Barcelona, C/ Martí i Franquès 1, \\ Barcelona E-08028, Spain \\ ${ }^{2}$ Theoretical Division, MS B268, Los Alamos National Laboratory, Los Alamos, New Mexico 87545
}

(Received 31 May 2001; revised manuscript received 13 November 2001; published 20 March 2002)

\begin{abstract}
The effect of Fock exchange on the periodic description of the geometrical structure, elastic constants, and electronic and magnetic properties of $\mathrm{NiO}$ is analyzed. Hybrid density functionals which combine a portion of "exact" Fock exchange with conventional local density approximation (LDA) or generalized gradient approximation (GGA) functionals remedy a number of serious inconsistencies with the traditional LDA or GGA descriptions of this prototypical "Mott" insulator. For example, the hybrid B3LYP functional (which mixes $\sim 20 \%$ Fock exchange with GGA functionals) introduces a significant insulating gap and yields antiferromagnetic Heisenberg coupling constants between Ni sites $\left(J_{2}\right)$ in semiquantitative agreement with experiment. Closer inspection shows that while the B3LYP orbital band gap is in excellent agreement with experiment, the magnitude of the antiferromagnetic coupling is overestimated by slightly more than $50 \%$. This has led us to examine a simplified model which combines Fock exchange with the LDA exchange and correlation functionals. This combination allows us to study the magnitude and nature of the band gap, the magnitude of the unpaired spin densities in the different magnetic phases, and the two most important magnetic coupling constants as a function of the fraction of Fock exchange included. It is concluded that $\sim 35 \%$ Fock exchange gives a reasonably balanced description of all properties, including structural parameters, magnetic form factors, the antiferromagnetic Ni-Ni exchange constant, and the character and magnitude of the band gap.
\end{abstract}

DOI: 10.1103/PhysRevB.65.155102

PACS number(s): 71.15.-m, 75.30.Et, 75.10.Jm

\section{INTRODUCTION}

Nickel oxide, $\mathrm{NiO}$, is a prototype of a highly correlated material which exhibits insulating character and antiferromagnetic order that remains even at rather high temperatures $T_{N}=523 \mathrm{~K} .{ }^{1}$ In addition, $\mathrm{NiO}$ displays a simple cubic $\mathrm{NaCl}$ crystal structure, although it suffers a rhombohedral distortion below $T_{N}$ due to an anisotropic contraction of the lattice induced by the exchange magnetostriction that increases as the temperature is lowered. ${ }^{1}$ The measured optical gap of $\mathrm{NiO}$ is $\sim 4.0 \mathrm{eV},{ }^{2}$ clearly indicating that it behaves as an insulator. For years, this insulating character has been rationalized in terms of a Mott-Hubbard picture. ${ }^{3}$ However, this physical picture has been questioned by the photoemission experiments and analysis of Zaanen, Sawatzky, and Allen. ${ }^{4}$ These authors report a somewhat larger value for the optical band gap of $4.3 \mathrm{eV}$ and show that charge-transfer excitations from the oxygen to the unfilled $d$ band of the Ni cations also play a role in determining the $\mathrm{NiO}$ band gap. In other words, the band gap is not solely determined by the $d-d$ Coulomb interactions, i.e., by the on-site electron-electron repulsion term commonly designated as $U$ in the Hubbard model Hamiltonian. A similar conclusion is reached by analysis of photoemission and inverse photoemission results. 5 The strongly correlated nature of the electrons in $\mathrm{NiO}$ and the importance of charge-transfer excitations in determining the magnitude of the band gap have been confirmed by the resonant photoelectron spectroscopy study of Tjernberg et al. ${ }^{6}$ Finally, it is worth pointing out that rather recent experiments show that the electronic structure is not significantly influenced by the magnetic order. ${ }^{7}$

From the theoretical point of view, both semiempirical and $a b$ initio cluster models as well as periodic approaches have been used to study the electronic structure of $\mathrm{NiO}$. The cluster models generally employ configuration interaction (CI) approaches and use either a semiempirical determination of the parameters ${ }^{4,8}$ or a fully $a b$ initio treatment of the cluster. These models have been successfully used since the late seventies to describe many details of the electronic structure of $\mathrm{NiO}$ including photoemission ${ }^{9}$ and optical spectra, ${ }^{8,10-13}$ magnetic coupling, ${ }^{14,15}$ and the character of the band gap. ${ }^{16}$ However, one must recognize that the cluster model approach has its own set of problems and must be accompanied with careful investigations of the convergence of the results as a function of cluster size, the manner in which the cluster is truncated, and the nature and appropriateness of the embedding procedure which links it to the remainder of the crystal. Here we must point out that the success of the cluster model approach contrasts with the difficulties encountered by periodic approaches to describe the essential electronic structure features even at a qualitative level. Certainly, the local density approximation (LDA) to density functional theory (DFT), widely used in solid-state physics, fails to describe $\mathrm{NiO}$ as an insulator and predicts it to be a metal. ${ }^{17,18}$ This deficiency of the LDA is not fully repaired by the generalized gradient approximation (GGA) to the exchange-correlation functionals. In fact, at the GGA level of theory the $\mathrm{NiO}$ band gap is still too small, indicating either a metal or a semiconducting behavior. ${ }^{19-21}$ It has been suggested that the difficulty of the LDA (and of the GGA) to properly describe narrow-band insulators is related to the insufficient cancellation of the self-interaction correction (SIC) inherent in the local exchange functional. The SIC-LDA introduces a qualitatively correct $(\sim 3 \mathrm{eV})$ gap in the spectrum and improves the magnitude of the magnetic moments and the value of the lattice constant in $\mathrm{NiO}^{22,23} \mathrm{An}$ approach 
which supplements the LDA with an effective on-site repulsion $U$ has also become popular. ${ }^{24,25}$ This approximation also improves the gap and lattice constant, ${ }^{23}$ but as usually implemented involves the introduction of two semiempirical parameters. ${ }^{25}$ Perhaps the most sophisticated of the postLDA approaches is the many-body GW approximation in which the electron self-energy is given as the product of the interacting Green's function $G$ times the dynamically screened Coulomb potential W. This method repairs the selfenergy correction in a more controlled, formally acceptable way. It successfully introduces a gap in $\mathrm{NiO}$, which in the self-consistent implementation of the theory is $\sim 3.7 \mathrm{eV},{ }^{26}$ in excellent agreement with experiment. It is interesting to note that the self-consistency condition is important, as the earlier, ${ }^{27}$ non-self-consistent implementation of the theory gives a gap which is significantly larger than experiment $(\sim 5.5 \mathrm{eV})$. The $\mathrm{GW}$ approximation also improves the magnetic moments and density of states relative to the LDA.

A common feature of the SIC-LDA and GW methods described above is an attempt to remove the improper selfinteraction correction present in the LDA. An alternative approach consists in making use of the exact cancellation between Coulomb and exchange terms in Hartree-Fock theory. Becke first followed this line in a series of papers exploring the effect of Hartree-Fock exchange in the DFT energies. ${ }^{28}$ This author has shown that for a large series of molecular systems, including a part of Fock exchange in the DFT energy drastically improves the calculated energies while preserving the accuracy of the LDA in predicting equilibrium structures and vibrational frequencies. A semiempirical fit to thermochemical data led Becke to propose an approach closely related to the now widely used hybrid B3LYP exchange-correlation functional. ${ }^{29}$ This approach is very similar to the GGA method (BLYP) that uses Becke's nonlocal exchange functional ${ }^{30}$ and the correlation functional given by Lee, Yang, and Parr ${ }^{31}$ based on the original work of Colle and Salvetti on the correlation factor. ${ }^{32,33}$ However, in the hybrid method three parameters are fitted to experiment, the optimum fit being found for $\sim 20 \%$ Fock exchange in the exchange functional. Surprisingly, the B3LYP hybrid functional is able to reproduce the thermochemistry of transition metal containing molecules, although no transition-metal compounds were included in the data set used in the fit. ${ }^{34-36}$ Later, Adamo and Barone suggested using a single empirical parameter only and proposed the B1LYP (and other similar hybrid approaches) with just $25 \%$ Fock exchange. ${ }^{37}$ This small fraction of Fock exchange would not be expected to remove the self-interaction correction. However, the success of the hybrid functionals would seem to point to its importance. The recent work on the "exact exchange" (EXX) method is also interesting in this regard as it precisely removes the self-interaction error. ${ }^{38-44}$ Interestingly, if pure Fock exchange is coupled with conventional local or semilocal correlation functionals, the agreement of the theory with experiment is generally worse. ${ }^{45-47}$ This must reflect the cancellation of errors inherent in the usual combination of conventional exchange and correlation functionals.

Despite its important impact in theoretical chemistry, the application of hybrid DFT to solid-state problems has been more limited mainly because of the difficulty in handling the exchange and Coulomb series at a high level of accuracy. This capability has now been implemented in the CRYSTAL code, ${ }^{48}$ and the first hybrid DFT (B3LYP) calculations with periodic boundary conditions ${ }^{49}$ are just beginning to appear. Of particular interest to us is the recent work by Bredow and Gerson $^{21}$ and Muscat et al., ${ }^{50}$ which shows that the B3LYP approximation significantly improves the description of the band gap in several semiconductors and insulators, the "Mott" insulators $\mathrm{MnO}, \mathrm{CoO}$, and $\mathrm{NiO}$ among them. Recently, periodic calculations using the hybrid B3LYP method have been reported for $\mathrm{La}_{2} \mathrm{CuO}_{4} \cdot{ }^{51}$ Note that this is within a "frozen-orbital" one-electron band structure approximation. The magnetic properties of these antiferromagnets have not yet been addressed by periodic hybrid functional calculations, but in earlier cluster work Martin and Illas pointed out the importance of Fock exchange on the magnetic coupling and magnetic moments of narrow-band insulators such as $\mathrm{La}_{2} \mathrm{CuO}_{4} .{ }^{52,53}$ Martin and Illas found that the B3LYP approach overcomes many of the problems of the LDA, but still predicts magnetic coupling constants nearly a factor of 2 larger than experiment. They found that a larger fraction of Fock exchange, $\sim 50 \%$, led to improved agreement with experiment. Because of the cluster nature of their approximation, they were not able to examine the band gap. The effect of the Fock exchange on the theoretical description of narrow-band insulators needs to be investigated in detail. In fact, improving the description of the magnetic coupling by increasing the amount of Fock exchange may or may not deteriorate the band gap and related electronic structure properties. This work studies this issue in the prototypical "Mott" insulator $\mathrm{NiO}$.

\section{COMPUTATIONAL APPROACHES}

In this paper we use both cluster and periodic approaches to unravel the most salient features of the electronic structure of $\mathrm{NiO}$ and in particular to explore the influence of the exchange-correlation functional in the calculated properties. The present cluster and periodic calculations make use of a local basis set of Gaussian-type orbitals (GTO's) and involve all electrons either in the cluster or in the atoms defining the unit cell. Basis sets of similar quality are used in the cluster and periodic calculations in order to provide a meaningful comparison. The accuracy of the different hybrid DFT methods is established by comparing calculated results obtained by using a particular hybrid approach either to experiment or to accurate quantum-chemical calculations. Thus the calculated cell parameters are compared to the experimental values of Bartel and Morosin ${ }^{1}$ and the elastic constants to the experimental values extrapolated at $T=0$ reported by Duplessis et al. ${ }^{54}$ The calculated band gap is compared to the well-established experimental value which lies in the 4.0$4.3 \mathrm{eV}$ interval. ${ }^{2,4}$ Likewise, magnetic coupling constants will also be compared with available experimental data. ${ }^{55-58}$ For the nearest-neighbor and next-nearest-neighbor magnetic coupling constants, the experimental values have an uncertainty of $\sim 2 \mathrm{meV}$. It has been recently shown that accurate quantum-chemical cluster model calculations are able to provide accurate predictions of these effective parameters. ${ }^{59-62}$ Therefore, cluster model calculations have also been per- 
formed in order to narrow the experimental range. Finally, the magnetic form factors are compared to the experimental reported by Alperin ${ }^{63,64}$ and to the unrestricted Hartree-Fock (UHF) cluster model calculations of Chang et al. ${ }^{65}$

It is worth pointing out that most of the results presented in the forthcoming section focus on the magnetic properties of $\mathrm{NiO}$, since they have not yet been investigated with the hybrid functionals using full periodic boundary conditions. We find results which are completely compatible with the earlier cluster work. ${ }^{52,53}$ The hybrid approximations simultaneously give a qualitatively correct description of the gap and magnetic coupling. On a more quantitative scale, we note that a somewhat larger component of Fock exchange, $\sim 35 \%$, gives improved agreement with the coupling constant at the price of a somewhat too large value for the oneelectron band gap.

Except in the obvious case of elastic constants and cell parameters, all calculations have been carried out using the experimental cubic lattice at low temperature with $a$ $=4.1705 \AA$ as determined by Bartel and Morosin. ${ }^{1}$

\section{A. Periodic approach}

The periodic Hartree-Fock, LDA, and several hybrid DFT calculations (vide infra) have been carried out by means of the CRYSTAL98 program package ${ }^{48}$ which develops the Bloch functions as a linear combination of atom-centered Gaussiantype orbital basis sets. ${ }^{66,67}$ The present all-electron basis sets have been specifically designed to describe the electronic structure of $\mathrm{NiO}^{68}$ Thus the $\mathrm{Ni}$ atomic basis contains, $1 \mathrm{~s}$, $4 s p$, and $2 d$ contracted GTO's obtained by means of a $8 / 6411 / 41$ contraction of the $(20 s, 12 p, 5 d)$ primitive Gaussian set. The oxygen basis set includes $1 s$ and $3 s p$ contracted GTO's obtained from a $(14 s, 6 p)$ primitive set and a $8 / 411$ contraction scheme. The cutoff threshold parameters ITOL 1-5 of CRYSTAL (Ref. 48) for Coulomb and exchange integral evaluations have been set to the 7,7,7,7, and 14 strict values, respectively. The integration in reciprocal space has been carried out using a $k$-space grid parameter of 8 yielding 65 points in the irreducible first Brillouin zone for the structure. Here we remark that the antiferromagnetic magnetic structure, hereafter referred to as AF2, needs a double cell of the simple ferromagnetic cell in the $F m 3 m$ space group. This AF2 magnetic phase is formed by ferromagnetic (111) planes alternating its spin in the [111] direction. An auxiliary magnetic double cell has been used to construct a different antiferromagnetic phase, hereafter referred to as AF1, consisting of ferromagnetic (100) planes alternating their spin in the [100] direction [see Eq. (2) below].

It is important to remark that the same atomic basis sets have been used for Hartree-Fock and the different DFT-based approaches. In the DFT calculations, even-tempered auxiliary basis sets for fitting the exchange-correlation potential were used. The nickel auxiliary basis set contains $12 s$-type functions with exponents between 0.1 and 6000.0, $3 d$-type functions with exponents between 0.25 and 6.0, and, finally, $3 \mathrm{~g}$-type functions with exponents between 0.45 and 3.3. Similarly, the oxygen auxiliary basis contains $14 s$-type Gaussian functions with exponents between 0.07 and 4000.0,
$1 p$-type Gaussian function with exponent $0.5,1 d$-type Gaussian function with exponent 0.5 , and $1 f$-type Gaussian function with exponent 0.5 . The numerical thresholds used to ensure the numerical convergence of the self-consistent-field (SCF) procedure were set to $10^{-6}$ a.u. for the one-electron eigenvalues and to $10^{-7}$ a.u. for the total energy. This set of thresholds for the Coulomb and exchange series, the integration in reciprocal space, and the total energy are much more stringent than the usual standard settings and have been chosen to avoid possible numerical problems. Test calculations show that forcing a higher numerical accuracy does not introduce any significant change in the calculated properties.

The main goal of this series of periodic calculations is to analyze the geometrical structure, elastic constants, band gap, spin densities, magnetic form factors, and magnetic coupling constants of $\mathrm{NiO}$ at the same time. Following previous work on magnetic coupling in several narrow-band insulators using the Hartree-Fock approximation, ${ }^{48,69,70}$ a double-cell procedure, as commented above, has been used to extract the important magnetic coupling constants. The use of the Ising Hamiltonian that considers the magnetic interactions between nearest- $\left(J_{1}\right)$ and next-nearest- $\left(J_{2}\right)$ neighbor magnetic $\mathrm{Ni}^{2+}$ centers permits to relate the energy of the FM, $\mathrm{AF} 1$, and AF2 magnetic phases to the magnetic coupling constants $J_{1}$ and $J_{2}$. Hence, considering the Ising Hamiltonian defined as

$$
H=-\mathbf{J}_{1} \sum_{\langle i j\rangle} S_{z i} S_{z j}-\mathbf{J}_{2} \sum_{\langle k l\rangle} \mathbf{S}_{z k} \mathbf{S}_{z l},
$$

where $S_{z i}$ stands for the $z$ component of total spin on the magnetic center $i$ and $\langle i j\rangle$ and $\langle k l\rangle$ indicate sum over first and second neighbors, respectively. It is easy to show that the energy differences between the FM, AF1, and AF2 magnetic phases per formula unit are given by the simple formulas

$$
\begin{gathered}
E(\mathrm{AF} 1)-E(\mathrm{FM})=8 J_{1}, \\
E(\mathrm{AF} 2)-E(\mathrm{FM})=6 J_{1}+6 J_{2} .
\end{gathered}
$$

The cell parameters have been determined for the ferroand antiferromagnetic phases and the elastic constants calculated numerically by fitting the energy with respect to the deformation of the cell following the procedure described by Dovesi et al. ${ }^{71,72}$ The magnetic form factors have been calculated as the Fourier transform of the ground-state antiferomagnetic spin density.

In order to obtain the relevant electronic structure parameters for the electronic antiferromagnetic ground state the same double-cell approach has been used to obtain the band gap and density of states. This procedure avoids any possible bias on the electronic structure arising from the choice of a ferromagnetic solution forced by the use of a single unit cell. Hence two $\mathrm{NiO}$ formula units in the primitive cell were used in the spin-unrestricted calculations aimed to describe the two antiferro- and the ferromagnetic phases relevant to magnetic coupling. We must point out again that most of the calculations have been carried out using two different double cells (vide infra), although in some particular cases only the most stable AF2 antiferromagnetic state with parallel spins in 
the (111) planes has been considered. Notice that previous periodic UHF (Ref. 68) and DFT (Refs. 17-21 and 50) calculations predict that AF2 is the magnetic ground state for $\mathrm{NiO}$. The same ground state is obtained using the present hybrid DFT approaches. Finally, we note that for the ferromagnetic state the energy per formula unit computed with the single or double cell does not differ within the numerical threshold for the SCF procedure.

A last point concerns the definition of the different hybrid exchange correlational functionals used in this work. Since the same functionals are used in the periodic and cluster calculations, we postpone this part and report the necessary information in Sec. IIC.

\section{B. Cluster model approach}

The cluster model approach has been used with a twofold purpose: on the one hand to show that the effect of the exchange-correlation functional in the description of the magnetic coupling is the same regardless of the materialcluster or periodic-model used and on the other hand to obtain an accurate prediction of the nearest-neighbor, $J_{1}$, and of the next-nearest-neighbor, $J_{2}$, magnetic coupling parameters. The cluster model contains a quantum-mechanical part and a proper representation of the rest of the crystal by means of a simple and convenient embedding procedure. As in previous researches, ${ }^{14,52,53,62,73-80}$ the quantummechanical part contains the two magnetic centers of interest and the anions in the proper coordination sphere. The final cluster models thus designed are $\mathrm{Ni}_{2} \mathrm{O}_{11}$ and $\mathrm{Ni}_{2} \mathrm{O}_{10}$ depending on whether the Ni-O-Ni magnetic path corresponds to an angle of $180^{\circ}$ (single bridge) or $90^{\circ}$ (double bridge). Total ion potentials (TIPs) ${ }^{81}$ and an array of point charges ${ }^{82,83}$ to account for the short-range and Madelung potential effects, respectively, surround both clusters.

Different kinds of calculations have been carried out for the embedded $\mathrm{NiO}$ cluster models. The first type of calculation is UHF for the ferromagnetic and antiferromagnetic spin states of the dimer in the cluster. We have to point out that strictly these two states are not spin eigenfunctions and, hence, do not have a defined multiplicity. Moreover, the antiferromagnetic state is necessarily a broken symmetry solution. Nevertheless, the ferromagnetic state is a good approximation to the high-spin state and the energy of the low-spin state can be quite easily derived from the energy corresponding to the broken-symmetry antiferromagnetic solution. ${ }^{52,53,80,84-86}$ In this way, the UHF value of the $J_{1}$ and $J_{2}$ coupling constants is obtained. Next, LDA and different hybrid DFT-based calculations described in detail in the next section have been carried out for the same ferro- and antiferromagnetic states to provide the DFT estimates of the same magnetic coupling constants. The reason for this set of calculations is that, at each level of theory, they can be directly compared with the periodic calculations.

The broken-symmetry solution provides only an estimate of the magnetic coupling constants. In order to obtain accurate values of both $J_{1}$ and $J_{2}$ explicitly correlated wave functions of increasing complexity were obtained using CI techniques and from their corresponding energies the magnetic coupling constants were obtained. The first level of theory is the complete active space self-consistent-field (CASSCF) approach. CASSCF (Ref. 87) calculations were carried out to obtain wave functions for the singlet, triplet, and quintet states arising from the coupling of the atomic ${ }^{3} A_{2 g}$ multiplet derived from the $\left(e_{g}\right)^{2}$ electronic configuration in each $\mathrm{Ni}^{2+}$ cation. Hence the CAS contains four electrons and four orbitals leading to a total of 36 states, although only the lowest singlet, triplet, and quintet states are relevant to magnetic coupling. Next, dynamical correlation effects have been included by means of two different approaches. The first one is second-order multireference perturbation (CASPT2) theory using the CASSCF wave functions as zero order. ${ }^{88,89}$ The second approach is the difference dedicated configuration interaction method with two (DDCI2) or three (DDCI3) degrees of freedom. ${ }^{90}$ For magnetic problems the DDCI2 should include all terms contributing to the magnetic coupling constant. ${ }^{90}$ However, it has been recently shown that when instantaneous relaxation of the charge-transfer excitations is important, the DDCI3 is necessary to achieve a quantitative agreement between theory and experiment. ${ }^{59,60}$ The molecular orbitals necessary to construct the DDCI configuration interaction wave function were obtained from a CASSCF calculation on the quintet state. Using this set of molecular orbitals, complete active space configuration interaction (CASCI) wave functions were obtained for the singlet, triplet, and quintet states and used as model spaces for the construction of the DDCI2 and DDCI3 spaces. The resulting DDCI2 and DDCI3 expansions contain up to 117354 and 7664252 determinants in $D_{2 h}$ symmetry, respectively. The expectation energy value corresponding to these expansions has been obtained variationally: i.e., by explicit diagonalization of the matrix representation of the hamiltonian in the CI space.

The UHF and DFT calculations explicitly consider all electrons in the cluster model and have been carried with the GAUSSIAN98 suite of programs. ${ }^{91}$ The Gaussian basis sets were $6-3111 \mathrm{G}^{*}$ for $\mathrm{Ni}$ and $6-31 \mathrm{G}^{*}$ for all $\mathrm{O}$ atoms. The CASSCF and CASPT2 calculations have been carried out by means of the MOLCAS4.0 package ${ }^{92}$ and the DDCI ones have been performed with the programs written by Maynau and Ben Amor $^{93,94}$ after a transformation ${ }^{95}$ of the molecular integrals obtained by MOLCAS. These calculations use the generally contracted atomic natural orbital (ANO) basis sets developed by various authors ${ }^{96-98}$ and internally stored in the MOLCAS package ${ }^{92}$ and are $[5 s 4 p 3 d]$ for $\mathrm{Ni},[4 s 3 p 1 d]$ for the bridging $\mathrm{O}$, and $[3 s 2 p]$ for the environmental $\mathrm{O}$ atoms. The cluster anions were surrounded by a Ni TIP, developed by Hay and used in previous work. ${ }^{52}$

\section{Description of the exchange-correlation functionals used}

Different hybrid functionals were used to investigate the effect of Fock exchange in the series of properties discussed above. In addition, UHF and LDA calculations were also performed for comparison. The same functionals were used in cluster and periodic calculations, thus providing a one-toone correspondence between both limiting descriptions of NiO. The first hybrid is the semiempirical B3LYP 
TABLE I. Results for the cell parameter of $\mathrm{NiO}$ ( $a$ in $\AA$ ) obtained from the periodic approach.

\begin{tabular}{cccccc}
\hline \hline Cell & UHF & Fock-50 & Fock-35 & B3LYP & Expt. \\
\hline FM & 4.264 & 4.156 & 4.144 & 4.232 & \\
AF2 & 4.261 & 4.152 & 4.144 & 4.227 & $4.1705^{\mathrm{a}}$ \\
\hline \hline
\end{tabular}

${ }^{a}$ Reference 1 .

functional, ${ }^{28}$ which, as commented above, contains $20 \%$ Fock exchange. Specifically, the B3LYP energy functional has the form

$$
\begin{aligned}
E_{\mathrm{xc}}^{\mathrm{B} 3 \mathrm{LYP}}= & (1-A) E_{x}^{\mathrm{Slater}}+A E_{x}^{\mathrm{HF}}+B E_{x}^{\mathrm{Becke}}+C E_{\mathrm{corr}}^{\mathrm{LYP}} \\
& +(1-C) E_{\mathrm{corr}}^{\mathrm{VWN}},
\end{aligned}
$$

where $E_{x}^{\text {Slater }}$ is the Dirac-Slater local exchange, $E_{x}^{\mathrm{HF}}$ the Hartree-Fock exchange, $E_{x}^{\text {Becke }}$ the gradient part of the Becke-gradient-corrected exchange functional, ${ }^{29} E_{\text {corr }}^{\mathrm{LYP}}$ the correlation functional of Lee, Yang, and Parr, ${ }^{31}$ and $E_{\text {corr }}^{\mathrm{VWN}}$ the local electron gas correlation functional of Vosko, Wilk, and Nusair, ${ }^{99} A, B$, and $C$ are the coefficients determined by Becke from a fit to experimental heats of formation for a benchmark test consisting of 55 first- and second-row molecules. The parameters $A, B$, and $C$ were determined to be $A=0.20, B=0.72$, and $C=0.81$. It should be noted that Becke did not use $E_{\text {corr }}^{\mathrm{LY}}$ in his fit, but rather the GGA correlation functional of Perdew et al. ${ }^{100,101}$ The B3LYP functional, as opposed to Becke's original functional, has been popularized by its availability in the GAUSSIAN suite of electronic structure codes. ${ }^{91}$ It has had a dramatic impact in quantum chemistry.

The other hybrid approach examined here mixes Fock and Dirac-Slater exchange functionals with the LDA correlation functional. By tuning the parameter $\delta$ between 0 and 1 , we can follow the progression from the pure LDA to pure HF exchange, always maintaining the LDA correlation part:

$$
E_{\mathrm{xc}}=(1-\delta) E_{x}^{\mathrm{Slater}}+\delta E_{x}^{\mathrm{HF}}+E_{\mathrm{corr}}^{\mathrm{LDA}} .
$$

The reason we choose the LDA for the remaining exchange and for the correlation contribution is that this permits us to clearly differentiate the effect of Fock exchange without having to refer to external parameters and to particular forms of the gradient-corrected functionals. In addition, it has been shown that the choice of the correlation functional on the calculated magnetic coupling constant is rather small and the same occurs for the DFT part of the exchange contribution. ${ }^{52,53}$ Among the several possible mixtures we report here results for $35 \%$ and $50 \%$ of Fock exchange, respectively, and denote these approaches as Fock-35 and Fock-50. Notice that the latter is close, but not identical to the Half-and-Half functional proposed by Becke. ${ }^{28}$

\section{RESULTS AND DISCUSSION}

In this section we report the results obtained for various properties of $\mathrm{NiO}$. In order to facilitate the discussion the contents of this section have been organized focusing on the different properties. Thus cell parameters and elastic con- stants are first described. The electronic structure is described in Sec. III B followed by a description of the magnetic coupling (Sec. III C), and, finally, Sec. III D is devoted to a discussion of the magnetic form factors.

\section{A. Cell parameters and elastic constants}

The equilibrium unit-cell parameters are those minimizing the total energy per unit cell. In the case of $\mathrm{NiO}$ the cubic symmetry leads to a single unit-cell parameter. This parameter has been determined using both FM and AF2 magnetic phases. However, no significant differences appear and the small discrepancies are basically due to numerical noise (Table I). The UHF and B3LYP results agree with the numbers given by Towler et al. ${ }^{68}$ and Bredow and Gerson. ${ }^{21}$ Both are larger than the experimental value by $1.5 \%$ and $2.2 \%$, respectively. LDA results are not reported since the difficulties in converging the magnetic phases give too large numerical noise in the fits. However, the general trend is that it tends to give an underestimation of the cell parameter $(\sim 4.08 \AA)$, in agreement with previous plane-wave calculations. ${ }^{20}$ The hybrid Fock- $X$ approaches give excellent agreement with experiment: i.e., $0.6 \%$ for Fock-35 and $0.3 \%$ for Fock-50. From this first set of results it can be concluded that all methods predict values of the cell parameter in good agreement with experiment, although the hybrid functionals seem to perform reasonably better.

The elastic constants are second derivatives of the energy per unit cell with respect to the elements of the infinitesimal Lagrangian strain tensor

$$
C_{i j}=\frac{1}{V} \frac{\partial^{2} E}{\partial \varepsilon_{i} \partial \varepsilon_{j}},
$$

where $V$ is the volume of the cell. In the present version of CRYSTAL the energy derivatives must be evaluated numerically. In order to avoid large numerical errors in the fitting procedure particular care is required in the selection of the computational parameters, of the points where the energy is evaluated, and of the numerical integration procedure needed in the DF calculations.

The calculation of the elastic constants involves deformations from the unit cell, and when this occurs the symmetry point group is reduced to a subgroup of the original point group. The new point group is automatically selected by the CRYSTAL code. Off-diagonal (partial derivatives) elastic constants can be computed as linear combinations of singlevariable energy curves. For the $\mathrm{NiO}$ case, there are only four independent elastic constants $B, C_{11}, C_{12}$, and $C_{44}$. Several strategies have been proposed to perform simple cell deformations that permit the calculation of the elastic constants conserving the maximum number of symmetry 
TABLE II. Calculated elastic constants and bulk modulus (in GPa) for NiO using the FM phase, compared with experimental data at $0 \mathrm{~K}$ (Ref. 54). The results in the first column correspond to calculations at constant volume and have been taken from Towler et al. (Ref. 68).

\begin{tabular}{lcccccc}
\hline \hline$C_{i j}$ & UHF $(\mathrm{V})$ & UHF & Fock-50 & Fock-35 & B3LYP & Expt. \\
\hline$C_{11}$ & 399 & 378 & 450 & 473 & 435 & 211 \\
$C_{11}-C_{12}$ & 272 & 257 & 301 & 335 & 285 & 90 \\
$C_{12}$ & 127 & 121 & 149 & 138 & 150 & 121 \\
$C_{44}$ & 121 & 147 & 129 & 119 & 114 & 109 \\
$B$ & 215 & 207 & 237 & 244 & 222 & 145 \\
$B=\left(C_{11}+2 C_{12}\right) / 3$ & 213 & 206 & 249 & 249 & 245 & 151 \\
\hline \hline
\end{tabular}

operators. ${ }^{48,71,72}$ Following the deformation of the unit cell, internal relaxation of the atoms may be necessary depending on the space-group symmetry. However, for the present case there are no internal parameters to optimize: all are fixed by symmetry. In this work deformations of up to $2 \%$ of the cell parameter have been considered. This is well within the limit of small deformations to use quadratic expansion of the total energy with respect to the strain tensor and use second-, third-, and fourth-order polynomial fits to the appropriate second derivatives at the energy minimum. The consistency between derivatives of the second-, third-, and fourth-order polynomials shows that the accuracy of the fits is sufficient since differences are less than $4 \%$. In addition, non-volumeconserving deformations give essentially the same results as those where the volume is preserved because $\Delta V$ is small. The deformations considered maintain at least eight symmetry operators. Present calculations ignore any dynamical contribution to the free energy due to lattice vibrations. Hence the theoretical results have to be compared to the $T=0 \mathrm{~K}$ extrapolation of the experimental results given by DuPlessis et al. ${ }^{54}$ The differences between FM and AF2 results are small for the simpler $C_{44}$ and $B$ parameters $(\sim 5 \%)$ and seem to be mainly due to numerical errors in the fits. There is no reason for a difference in the rest of the elastic constants: consequently, only calculations of the FM phase are reported.
This insensitivity with respect to the magnetic state is in line with the results reported by Towler et al. ${ }^{68}$ in their UHF calculations.

The calculated results for elastic constants and bulk modulus are reported in Table II. It must be pointed out that as noted by Towler et al. ${ }^{68}$ the wide variation in the published experimental data at different temperatures ${ }^{54,102,103}$ precludes a detailed comparison. Overall, none of the methods produce satisfactory results and it can be concluded that all the Hamiltonians largely overestimate $B$ and $C_{11}$, which are related to $\mathrm{Ni}-\mathrm{O}$ distances, whereas they provide reasonable results for $C_{12}$ and $C_{44}$ basically related to bond angles. Clearly, elastic constants do not provide a useful way to decide about the performance of the different hybrid functionals.

\section{B. Electronic structure}

The nature of the chemical bond in bulk $\mathrm{NiO}$ has been described to be largely ionic with net charges on $\mathrm{Ni}$ and $\mathrm{O}$ close to the full ionic character limit. $8,10,12,68,104$ Not unexpectedly, the present results indicate that the qualitative picture of the chemical bond in bulk NiO is strongly dependent on the particular method used to describe its electronic structure (Table III). Judging from the Mulliken charges, the bonding appears to be almost completely ionic at the UHF

TABLE III. Results for electronic-structure-related parameters of $\mathrm{NiO}$ obtained from the periodic approach using the experimental cell parameters. The calculated values are the indirect band gap $(\Delta)$, the total unpaired spin density on $\mathrm{O}\left(\mu_{\mathrm{O}}\right)$ and $\mathrm{Ni}\left(\mu_{\mathrm{Ni}}\right)$ from the Mulliken analysis, the net charge on $\mathrm{Ni}\left(\sigma_{\mathrm{Ni}}\right)$ also from Mulliken analysis, and the magnetic coupling along the $90\left(J_{1}\right)$ and $180\left(J_{2}\right)$ Ni-O-Ni magnetic path. The experimental magnetic moments on $\mathrm{Ni}$ are within the (1.6-1.9) $\mu_{B}$ range (Refs. 63-56).

\begin{tabular}{ccccccc}
\hline \hline & UHF & Fock-50 & Fock-35 & B3LYP & LDA & Expt. \\
\hline$\Delta(\mathrm{eV})$ & 15.1 & 8.4 & 6.2 & 4.1 & 0.0 & $\sim 4.0^{\mathrm{a}}$ \\
$\mu_{\mathrm{Ni}}(\mathrm{AF} 2)$ & 1.91 & 1.81 & 1.75 & 1.67 & 1.18 & \\
$\mu_{\mathrm{Ni}}(\mathrm{FM})$ & 1.92 & 1.82 & 1.77 & 1.73 & 1.57 & \\
$\mu_{\mathrm{O}}(\mathrm{FM})$ & 0.08 & 0.18 & 0.24 & 0.27 & 0.44 & \\
$\sigma_{\mathrm{Ni}}(\mathrm{AF} 2)$ & 1.87 & 1.76 & 1.72 & 1.66 & 1.49 & \\
$J_{1}(\mathrm{meV})$ & 0.8 & 1.5 & 1.9 & 2.4 & 11.9 & $<+1.4^{\mathrm{b}, \mathrm{c}}$ \\
$J_{2}(\mathrm{meV})$ & -4.6 & -10.6 & -19.7 & -26.7 & -71.3 & {$[-19.8,-17.0]^{\mathrm{b}, \mathrm{c}}$} \\
\hline \hline
\end{tabular}

${ }^{a}$ Reference 2 .

${ }^{\mathrm{b}}$ Reference 57.

${ }^{\mathrm{c}}$ Reference 58 . 

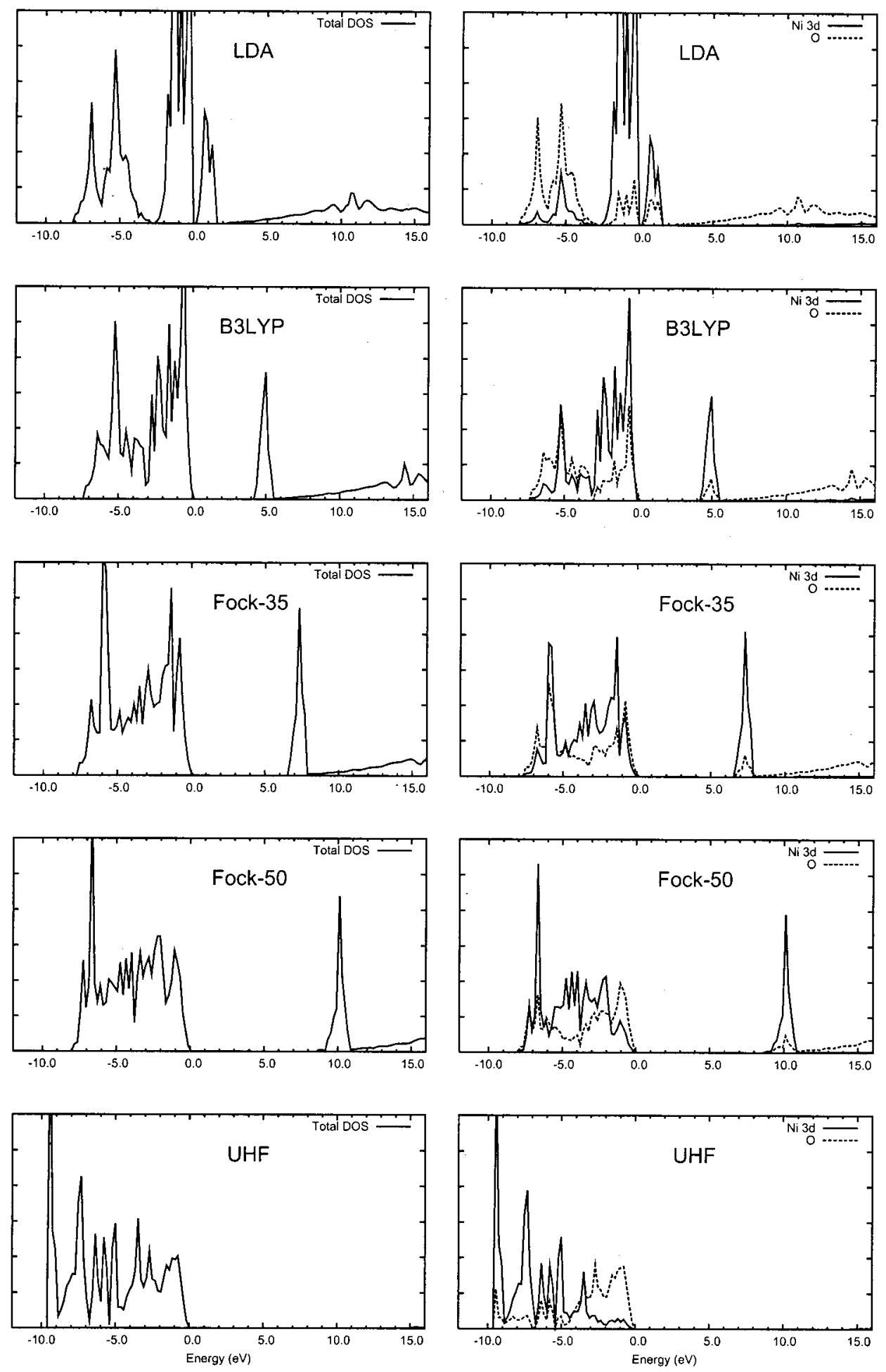

FIG. 1. Total and projected density of states (DOS) on Ni and O basis sets.

level, whereas it is strongly covalent in DFT or even metallic in the LDA. This is confirmed by the density of states (DOS) represented in Fig. 1. Clearly, this dependence on the qualitative description of the chemical character of $\mathrm{NiO}$ leads to a similar dependence on the prediction of the different electronic structure properties. Thus UHF predicts the correct insulating character, but the band gap is much too large. As is well known, the LDA predicts $\mathrm{NiO}$ to be a metal rather than an insulator (Table III). Depending on the amount of Fock exchange, the band gap varies from $15.1 \mathrm{eV}$ to almost zero with the B3LYP method predicting a band gap in excellent agreement with experiment and with the previous B3LYP 
calculations. ${ }^{50}$ Further inspection of the results reported in Table III shows that B3LYP predicts a magnetic coupling constant-determined by projecting a pure spin function from the broken-symmetry antiferromagnetic state and comparing that energy with the energy of the high-spin ferromagnetic state-which is nearly twice as large as experiment. This quantity also presents a marked dependence on the exchange component as expected from the work of Martin and Illas on cluster models of similar compounds. ${ }^{52,53}$ The UHF prediction for the magnetic coupling constant is too small, and that for the LDA is too large. In fact, since the LDA yields a metal, the approach used to extract the coupling constant is more reflective of an effective hopping integral. ${ }^{52,53}$ In addition, note that the LDA is the only method predicting a large difference in the unpaired spin density of $\mathrm{Ni}$ in the ferro- and antiferromagnetic states. This contradicts experimental evidence that shows $\mathrm{NiO}$ to be a clear example of a Heisenberg system and it also conflicts with the experimental measurements of Tjernberg et al. ${ }^{7}$ If we take the amount of Fock exchange as a semiempirical parameter, we conclude that Fock-35 provides a rather reasonable description of the magnetic coupling. The band gap in this approximation is now significantly larger than experiment, $\sim 6 \mathrm{eV}$ vs the $4.0-4.3 \mathrm{eV}$ experimental gap. However, we stress that this is the band gap obtained from the oneelectron description. The band gap obtained in a HartreeFock calculation is even larger because the virtual orbitalsi.e., the conduction band - are obtained in the Coulomb and exchange fields of the total number of electrons in the unit cell. Consequently, the orbital energies are too high as compared to excitation energies. In molecular systems $\Delta \mathrm{SCF}$ calculations usually provide better estimates, but this technique cannot be applied in periodic calculations. The situation in DFT is more delicate because the Kohn-Sham determinant only provides a reference system with an electron density equal to the exact ground-state electron density of the system. The physical meaning of electron energies, except for Janak's theorem about the highest-occupied orbital, is less clear. Therefore, it is not too disturbing that the Fock-35 band gap is too large.

One other interesting point in Table III is that all DFTbased methods seem to have a common shortcoming. All predict a significant spin density on oxygen in the ferromagnetic state. We are aware of no experimental evidence supporting this prediction. The reduction of the unpaired spin on the $\mathrm{O}$ bridge site as one increases the fraction of Fock exchange is once again a reflection of the reduction in the covalent character as the UHF limit is approached. The tendency of DFT-based methods to exceedingly delocalize electron density on the ligands bridging the magnetic centers has been recently discussed by Chevreau et al. ${ }^{105}$ for a series of systems. These include $\mathrm{La}_{2} \mathrm{CuO}_{4}$, which, in a way, is similar to $\mathrm{NiO}$ : both are antiferromagnetic systems for which the LDA predicts a metallic ground state. Chevreau et al. have found that the failure of the LDA to describe the magnetic coupling is due to the too strong delocalization that leads to a qualitatively incorrect electron density in the re- gion near the nuclei. These authors have also shown that gradient-corrected and hybrid functionals correct this defect, but in an exaggerated way. ${ }^{105}$

Next, we turn our attention to the picture of the electronic structure arising from the projected and total density of states obtained at the various levels of theory (Fig. 1). First of all, we remind the reader that the density of states based on the results of spin-polarized Hartree-Fock or density functional calculations must be taken with a grain of salt and provide a qualitative point of view only. This is because as opposed to restricted Hartree-Fock calculations where Koopmans' theorem holds, there is no direct mathematical relationship between the one-electron levels issued from a spin-polarized HF or DFT calculation and the excitation energies except for the highest-occupied level. ${ }^{106-108}$ Nevertheless, there are numerical studies of the eigenvalues of near-exact Kohn-Sham effective potentials which show that Kohn-Sham eigenvalue differences are surprisingly good estimates of excitation energies. ${ }^{109,110}$ The situation in UHF is slightly worse because the potential felt by occupied and virtual orbitals is different contrary to what happens in DFT. A thoughtful discussion about the physical meaning of Kohn-Sham and Hartree-Fock eigenvalues has been recently given by Baerends and Gritsenko. ${ }^{111}$ Before leaving this point, we also acknowledge the fact that such analyses are common practice and often agree well with photoemission results. In principle, however, an approximation which explicitly considers the final $N-1$ electron system, such as is done in the GW approximation, is on much firmer ground.

The projected density of states of $\mathrm{NiO}$ shows a dramatic variation with the amount of Fock exchange, especially in the Ni $d$-like bands. All hybrid approaches predict the peaks arising from $\mathrm{Ni} d$-like bands and the $\mathrm{O} 2 p$-like bands to overlap in energy and are markedly different from the LDA where a clear and unphysical separation between $\mathrm{Ni}(3 d)$ and $\mathrm{O}(2 p)$ peaks shows up. The proximity between the peaks arising from $\mathrm{Ni} d$-like bands and $\mathrm{O} 2 p$-like bands is in agreement with experiment and points to the difficulty with describing $\mathrm{NiO}$ either as a Mott-Hubbard or as a chargetransfer insulator on the basis of the one-electron band structure alone. This proximity in energy does not necessarily imply covalent bonding: the $\mathrm{Ni}$ and $\mathrm{O}$ bands are perfectly distinguishable. The LDA description predicts a single and broad Ni peak containing all the different $d$ one-electron levels without a clear distinction between occupied and virtual bands, i.e., a near-metallic state, with a strong mixing of the states arising from the $t_{2 g}$ and $e_{g}$ manifolds. Thus the gap in the LDA is mainly of $d$ - $d$ character in contradiction with the experimental evidence for an $\mathrm{O}(2 p) \rightarrow \mathrm{Ni}(3 d)$ nature. $^{5-7}$ The introduction of Fock exchange generates a noticeable gap which increases from B3LYP to UHF. In addition, the nature of the band gap changes. With increasing Fock exchange, the $d$-character decreases at the Fermi energy and is pushed "down" to higher binding energy. Simultaneously, the $\mathrm{O}(2 p)$ density at the Fermi energy increases. The unoccupied density of states is $d$ like in all approximations, a simple reflection that $\mathrm{NiO}$ is nearly ionic and the $\mathrm{O}(2 p)$ 
TABLE IV. Unrestricted Hartree-Fock and spin-polarized DFT results for NiO from the cluster approach using the experimental cell parameters. The unpaired spin density on the cluster central $\mathrm{O}$ atom $\left(\mu_{\mathrm{O}}\right)$ and on the $\mathrm{Ni}\left(\mu_{\mathrm{Ni}}\right)$ centers arises from Mulliken analysis, and the magnetic couplings along the $90^{\circ}$ and $180^{\circ}$ Ni-O-Ni magnetic paths ( $J_{1}$ and $J_{2}$, respectively) have been obtained by means of the broken-symmetry approach. References for the relevant experimental data are included in the footnote of Table III.

\begin{tabular}{lcccccc}
\hline \hline & UHF & Fock-50 & Fock-35 & B3LYP & LDA & Expt. \\
\hline$\mu_{\mathrm{Ni}}(\mathrm{AF} 2)$ & 1.91 & 1.82 & 1.77 & 1.70 & 1.49 & \\
$\mu_{\mathrm{Ni}}(\mathrm{FM})$ & 1.93 & 1.82 & 1.78 & 1.73 & 1.59 & \\
$\mu_{\mathrm{O}}(\mathrm{FM})$ & 0.03 & 0.05 & 0.06 & 0.08 & 0.12 & \\
$J_{1}(\mathrm{meV})$ & 1.0 & 2.2 & 2.4 & 3.2 & 8.7 & $<+1.4$ \\
$J_{2}(\mathrm{meV})$ & -5.4 & -12.9 & -21.9 & -29.2 & -93.6 & {$[-19.8,-17.0]$} \\
\hline \hline
\end{tabular}

levels therefore nearly full. Thus, not only is the magnitude of the gap improved, but it becomes more charge transfer in character as well.

As mentioned in the Introduction, there have been other approaches which attempt to fix the incorrect description of the LDA band gap, orbital character, and spin densities in $\mathrm{NiO}$. In particular, we quote the $\mathrm{LDA}+U,{ }^{25} \mathrm{LDA}+\mathrm{SIC},{ }^{22}$ and LDA+GW (Refs. 26 and 27) approaches. Massida et $a l .{ }^{26}$ report a detailed comparison of these three techniques. The three different corrections to the LDA are able to predict a reasonable value for the band gap and magnetic moments, although the latter are generally smaller than the smallest experimental value. However, while LDA+ SIC is able to open a gap with the oxygen levels near the Fermi energy, it still predicts a clear and wrong separation between the $\mathrm{Ni}(3 d)$ and $\mathrm{O}(2 p)$ peaks.

\section{Magnetic coupling}

Our results for the magnetic moments and coupling constants have already been discussed in the previous section and reported in Table III. From the discussion in Sec. III A it appears that all hybrid approaches employed in the present work provide spin densities which are almost the same for the ferro- and antiferromagnetic phases, in agreement with experiment. All methods, including the LDA, are able to correctly predict that the AF2 antiferromagnetic state with parallel spins in the (111) planes is the $\mathrm{NiO}$ electronic ground state, in agreement with experiment. ${ }^{7}$ However, it is also worth mentioning that the LDA representation of the ferromagnetic state is also metallic, indicating how dangerous it is to extract conclusions from a part of the information only.

Once the magnetic ground state has been obtained, it is possible to compute the $J_{1}$ and $J_{2}$ magnetic coupling constants by exploring different magnetic phases and using the pertinent energy differences described in Sec. II A. First, we note that except for the LDA all methods predict $\mathrm{NiO}$ to behave as a Heisenberg system, the difference in spin density for the different magnetic phases being negligible. The hybrid functionals predict spin densities that are in good agreement with the experimental magnetic moment. However, it is worth pointing out that the relationship between unpaired spin density and magnetic moment is not straightforward and will not be further discussed. As pointed out above, the magnetic coupling constants show a large variation with the amount of Fock exchange. Accurate values for the magnetic coupling constants can be obtained by means of explicitly correlated cluster model wave functions that indeed are spin eigenfunctions. There is a considerable body of literature that supports the idea that the magnetic coupling constant is a local quantity and can be accurately described by means of an embedded-cluster model and high-quality $N$-electron wave functions. ${ }^{59,62,70,78-80,112}$ Nevertheless, we find it appropriate to report in Table IV the calculated moments, $J_{1}$ and $J_{2}$ values predicted by means of an embedded-cluster model using the broken-symmetry approach with our set of functionals. The agreement between periodic and cluster calculations is almost perfect, strongly supports the conclusions reached in previous works, and fully justifies the use of a cluster model to study the magnetic coupling in NiO. Notice, however, that the spin density for oxygen in the ferromagnetic phase predicted by the DFT-based methods is noticeable (Table IV) and larger than the one encountered in the cluster calculations (Table III). This difference arises from the tendency of DFT methods to exaggeratedly delocalize the electron density near the nuclei and from the fact that this delocalization is not possible in the cluster because of the reduced coordination.

Table $\mathrm{V}$ collects the results for $J_{1}$ and $J_{2}$ in $\mathrm{NiO}$ using clusters with the CI approximations. The CASCI description corresponds to the Anderson superexchange model and predicts coupling constants with the right sign, but which are much too small. In addition, CASCI is very close to UHF as

TABLE V. Results for the magnetic coupling constants $J_{1}$ and $J_{2}$ of $\mathrm{NiO}$ from the cluster approach using the experimental cell parameters and various CI approaches.

\begin{tabular}{lcccccc}
\hline \hline & CASCI & CASSCF & CASPT2 & DDCI2 & DDCI3 & Expt. \\
\hline$J_{1}(\mathrm{meV})$ & +0.4 & +0.5 & +1.2 & +1.2 & +1.8 & $<+1.4$ \\
$J_{2}(\mathrm{meV})$ & -4.4 & -5.0 & -16.7 & -12.6 & -16.3 & {$[-19.8,-17.0]$} \\
\hline \hline
\end{tabular}


TABLE VI. Calculated magnetic form factors for $\mathrm{NiO}$ compared with experimental data of Alperin (Ref. 63) (rightmost column) for the AF2 phase. The values in this table correspond to the ones represented in Fig. 2 except for the experimental values where the experimental values have been scaled.

\begin{tabular}{lccccccc}
\hline \hline$h k l$ & $\sin \theta / \lambda$ & UHF $^{\mathrm{a}}$ & UHF & Fock-50 & Fock-35 & B3LYP & Expt. \\
\hline 111 & 0.1038 & 0.870 & 0.8783 & 0.8312 & 0.8017 & 0.7660 & $0.920 \pm 0.0250$ \\
$\overline{3} 11$ & 0.1988 & 0.765 & 0.7493 & 0.7075 & 0.6822 & 0.6515 & $0.828 \pm 0.0235$ \\
$\overline{1} 33$ & 0.2613 & 0.620 & 0.6223 & 0.5852 & 0.5635 & 0.5375 & $0.690 \pm 0.0175$ \\
511 & 0.3115 & 0.535 & 0.5668 & 0.5385 & 0.5217 & 0.5002 & $0.645 \pm 0.0150$ \\
333 & 0.3115 & 0.515 & 0.5073 & 0.4750 & 0.4562 & 0.4340 & $0.584 \pm 0.0165$ \\
$\overline{5} 33$ & 0.3931 & 0.355 & 0.3640 & 0.3430 & 0.3310 & 0.3162 & $0.438 \pm 0.0150$ \\
$\overline{7} 11$ & 0.4281 & 0.370 & 0.3698 & 0.3427 & 0.3282 & 0.3120 & $0.439 \pm 0.0200$ \\
155 & 0.4281 & 0.310 & 0.3230 & 0.3070 & 0.2977 & 0.2860 & $0.400 \pm 0.0100$ \\
$\overline{3} 55$ & 0.4604 & 0.295 & 0.2489 & 0.2356 & 0.2281 & 0.2189 & $0.329 \pm 0.0110$ \\
733 & 0.4907 & 0.230 & 0.2231 & 0.2046 & 0.1949 & 0.1841 & $0.300 \pm 0.0145$ \\
555 & 0.5191 & 0.150 & 0.1578 & 0.1501 & 0.1459 & 0.1407 & $0.243 \pm 0.0125$ \\
911 & 0.5461 & 0.275 & 0.2803 & 0.2660 & 0.2575 & 0.2472 & $0.358 \pm 0.0100$ \\
$\overline{9} 33$ & 0.5964 & 0.160 & 0.1630 & 0.1537 & 0.1485 & 0.1423 & $0.260 \pm 0.0125$ \\
$\overline{1} 77$ & 0.5964 & 0.095 & 0.0949 & 0.0853 & 0.0806 & 0.0756 & $0.170 \pm 0.0100$ \\
$\overline{7} 55$ & 0.5964 & 0.070 & 0.0711 & 0.0653 & 0.0627 & 0.0599 & $0.160 \pm 0.0100$ \\
377 & 0.6201 & 0.060 & 0.0573 & 0.0500 & 0.0466 & 0.0430 & $0.118 \pm 0.0075$ \\
$\overline{1} 111$ & 0.6648 & 0.200 & 0.1976 & 0.1851 & 0.1778 & 0.1692 & $0.255 \pm 0.0110$ \\
$\overline{5} 77$ & 0.6648 & 0.010 & 0.0086 & 0.0054 & 0.0045 & 0.0038 & $0.080 \pm 0.0100$ \\
955 & 0.6861 & 0.030 & 0.0348 & 0.0331 & 0.0325 & 0.0317 & $0.120 \pm 0.0125$ \\
1133 & 0.7067 & 0.115 & 0.1137 & 0.1057 & 0.1012 & 0.0958 & $0.165 \pm 0.0125$ \\
777 & 0.7268 & 0.000 & 0.0362 & 0.0368 & 0.0364 & 0.0356 & $0.035 \pm 0.0305$ \\
199 & 0.7653 & 0.010 & 0.0076 & 0.0073 & 0.0073 & 0.0074 & $0.050 \pm 0.0140$ \\
1311 & 0.7839 & 0.160 & 0.1607 & 0.1530 & 0.1478 & 0.1413 & $0.242 \pm 0.0250$ \\
$\overline{1} 155$ & 0.7839 & 0.015 & 0.0141 & 0.0126 & 0.0120 & 0.0115 & $0.137 \pm 0.0150$ \\
\hline 399 & 0.7839 & 0.015 & 0.0139 & 0.0131 & 0.0123 & 0.0113 & $0.030 \pm 0.0295$ \\
\hline
\end{tabular}

${ }^{a}$ The UHF cluster values are those previously reported by Chang et al. (Ref. 65).

expected from the fact that UHF includes a part of the nondynamical correlation effects. It is also important to realize that CASCI and CASSCF provide almost the same results. Since the only difference between CASCI and CASSCF is that the former uses a unique set of molecular orbitals and the latter uses self-consistent orbitals for each electronic state, it follows that the orbital part does not play a crucial role in the magnetic coupling. This is in full agreement with the experimental observation that the spin density is not sensitive to the magnetic order ${ }^{7}$ and with the fact that $\mathrm{NiO}$ can be described as a nearest-neighbor Heisenberg system. Inclusion of dynamical correlation effects largely improves the prediction of the magnetic coupling constants. Earlier research dealing with a broad family of ionic insulators suggests that the CI approximation known as DDCI3 provides values that are very close to available experimental data and provided reliable predictions for the as-yet experimentally unknown magnetic coupling constants. ${ }^{59,60}$ The DDCI3 value for $J_{2}$ in $\mathrm{NiO}$ suggests the lower limit of the experimental range as the most likely value. The agreement between DDCI3 and experiment is also consistent with our earlier conclusions regarding the hybrid functionals.

\section{Magnetic form factors}

The static structure factors of a crystal corresponds to the Fourier transform of the ground-state (charge or spin) density of the system. These can be determined experimentally after taking into account several corrective terms related to the thermal and zero-point motion of nuclei. These parameters are usually known as form factors and can be obtained from

$$
F_{\vec{k}}=\int \rho(\vec{r}) e^{i \vec{k} \cdot \vec{r}} d \vec{r}
$$

The $F_{\vec{k}}$ values are given in a relative value with respect to the total number of electrons described by the density $\rho(\vec{r})$ and the Miller indices $h, k, l$ refer to the relevant conventional cell. In the case of dealing with the spin density the Fourier transform in Eq. (6) provides the so-called magnetic form factor and the Miller indices $h, k, l$ refer to the conventional magnetic cell that doubles the crystalline one. In the present study the form factor has been calculated from the Fourier transform of the ground-state AF2 spin density and special care must be taken to relate the Miller indices of the conventional magnetic cell with those of the smaller primitive cells used in the calculations including the irreducible atoms. ${ }^{113}$

Table VI presents a summary of results and compares the form factors obtained by the different approaches with available data from experiment. Following Chang et al., ${ }^{65}$ we have scaled the experimental value to best fit each theoretical set of results. This is reasonable since the data reported by Alperin $^{63}$ are put in an absolute value relative to an external 

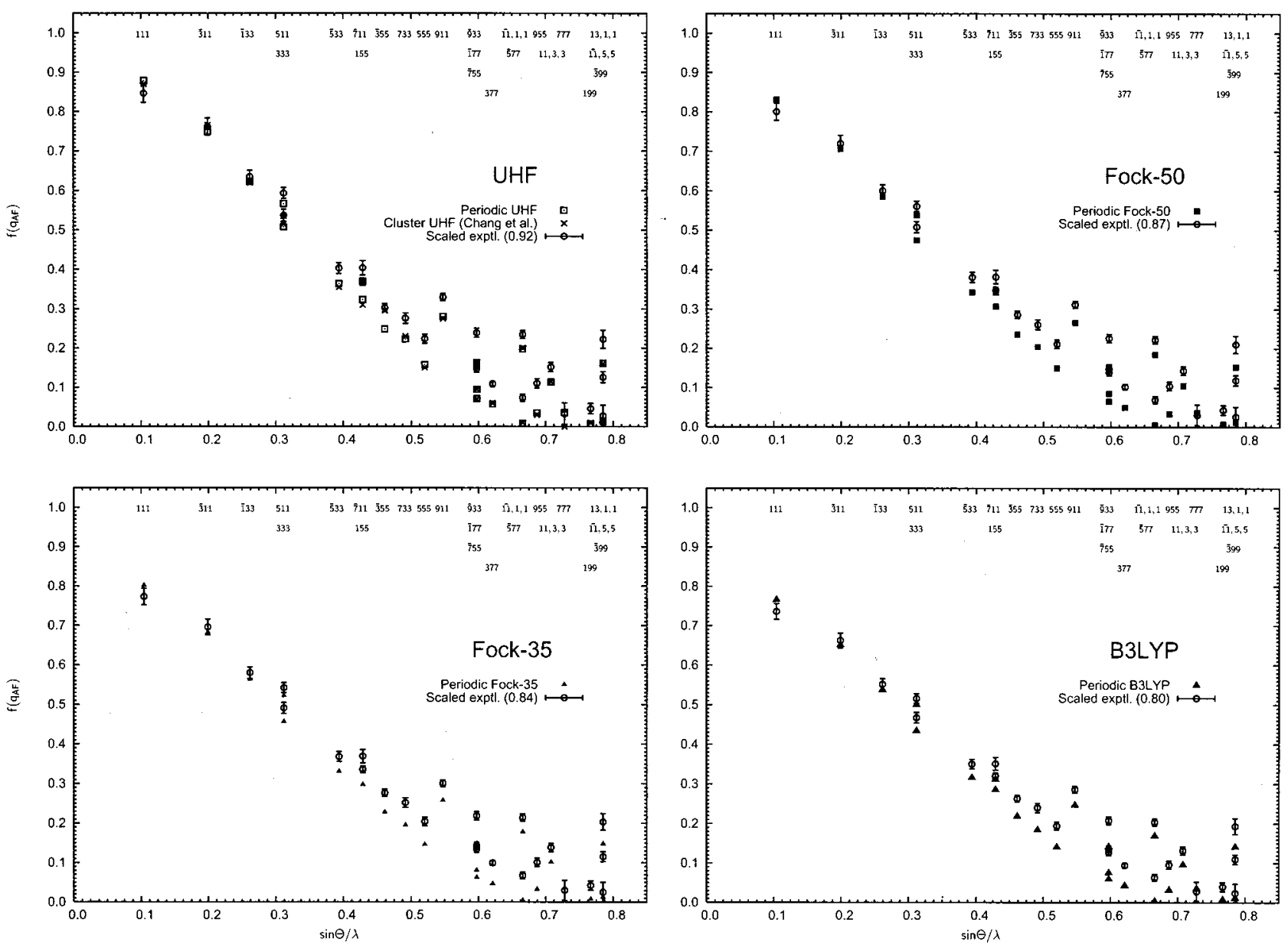

FIG. 2. Magnetic form factors of NiO obtained at the UHF, Fock-50, Fock-35, and B3LYP levels of theory. Scaled experimental values of Alperin (Ref. 63) and cluster calculations of Chang et al. (Ref. 65) are included for comparison.

experiment for the $(1,1,1)$ peak and scaling the rest of the peaks. The performance of the different methods is better analyzed by means of the graphical representation reported in Fig. 2. Here the situation is similar to that described for the cell parameter. All approaches follow the experimental trend without exhibiting large variations as a function of the exchange functional used.

\section{CONCLUSIONS}

The inclusion of Fock exchange has dramatic consequences on the electronic and magnetic coupling of $\mathrm{NiO}$. However, the effect on cell parameters, elastic constants, and magnetic form factors is much smaller. Comparing the magnitude and nature of the band gap, the magnitude of the unpaired spin densities in the different magnetic phases, and the two most important magnetic coupling constants has assessed the reliability of different functionals and allowed us to study these results as a function of the fraction of Fock exchange included in the wave function. A very important outcome of the present study is that none of the standard methods of electronic structure is able to provide results in agreement with experimental data for all properties. As is well known, the LDA has special difficulties with these "Mott-Hubbard" insulators. The introduction of Fock exchange into the LDA description has dramatic consequences on the electronic structure and magnetic properties. The widely used B3LYP hybrid functional leads to a qualitatively reasonable description of the electronic properties and a rather accurate value of the band gap, but the dominant antiferromagnetic coupling constant is predicted to be nearly $50 \%$ too large. Note also that the good agreement with the experimental gap may be misleading. It is empirically determined that a functional with $\sim 35 \%$ Fock exchange gives good agreement with the coupling constant $J_{2}$, an improved description of the charge-transfer nature of the band gap, and a magnitude for the gap in the orbital approximation of $\sim 6$ $\mathrm{eV}$, significantly higher than experiment, but perhaps not unreasonable. Interestingly enough, this improvement of Fock-35 on these properties with respect to the rest of methods does not significantly affect other properties such as cell parameter, elastic constants, and magnetic form factor.

A detailed comparison between periodic and cluster models shows that the magnetic coupling constant and unpaired spin densities (or magnetic moments) of $\mathrm{NiO}$ are local properties, the properties computed with identical functionals be- 
ing nearly the same when evaluated in a cluster or with periodic boundary conditions. This allowed us to use accurate configuration interaction wave functions to obtain a reliable theoretical estimate of $J_{2}$. It is concluded that the lowest experimental value for this physical property is the most likely.

To summarize, hybrid DFT approaches dramatically improve the qualitative and quantitative predictions of conventional DFT for the electronic and magnetic properties of $\mathrm{NiO}$. We expect that the conclusions reached in the present study will be of general validity and will hold for other strongly correlated systems as well. In conclusion, hybrid DFT represents a reliable alternative to those approaches aiming to correct the LDA and we believe provides impor- tant clues for the development of new and more accurate exchange-correlation functionals.

\section{ACKNOWLEDGMENTS}

This work has been financed by the DGICYT of the Spanish Ministerio de Educación y Ciencia (Project No. PB981216-CO2-01), in part by the CIRIT of the Generalitat de Catalunya (Grant No. SGR99-40), the Department of Energy and the University of California (Contract No. W-7405ENG-3G), and the LDRD program at Los Alamos National Laboratory. I. de P.R.M. is grateful to the University of Barcelona for support and thanks the Los Alamos National Laboratory for financial support during his stay. The authors wish to thank Prof. M. Catti and Prof. R. Dovesi for helpful discussions.
${ }^{1}$ L. C. Bartel and B. Morosin, Phys. Rev. B 3, 1039 (1971).

${ }^{2}$ R. J. Powell and W. E. Spicer, Phys. Rev. B 2, 2182 (1970).

${ }^{3}$ B. H. Brandow, Adv. Phys. 26, 651 (1977), and references therein.

${ }^{4}$ J. Zannen, G. A. Sawatzky, and J. W. Allen, Phys. Rev. Lett. 55, 418 (1985); G. A. Sawatzky and J. W. Allen, ibid. 53, 2339 (1984).

${ }^{5}$ S. Hüfner and T. Riesterer, Phys. Rev. B 33, 7267 (1986).

${ }^{6}$ O. Tjernberg, S. Söderholm, U. Karlsson, G. Chiaia, M. Qvarford, H. Nylén, and I. Lindau, Phys. Rev. B 53, 10372 (1996).

${ }^{7}$ O. Tjernberg, S. Söderholm, G. Chiaia, R. Girard, U. Karlsson, H. Nylén, and I. Lindau, Phys. Rev. B 54, 10245 (1996).

${ }^{8}$ A. Fujimori and F. Minami, Phys. Rev. B 30, 957 (1984).

${ }^{9}$ P. S. Bagus, Broer, R. de Graaf, and W. C. Nieuwpoort, J. Electron Spectrosc. Relat. Phenom. 99, 303 (1999).

${ }^{10}$ P. S. Bagus and U. Wahlgren, Mol. Phys. 33, 641 (1977).

${ }^{11}$ A. Fujimori, F. Minami, and S. Sugano, Phys. Rev. B 29, 5225 (1984).

${ }^{12}$ E. Lorda, F. Illas, and P. S. Bagus, Chem. Phys. Lett. 256, 377 (1996).

${ }^{13}$ C. de Graaf, R. Broer, and W. C. Nieuwpoort, Chem. Phys. 208, 35 (1996).

${ }^{14}$ C. de Graaf, F. Illas, R. Broer, and W. C. Nieuwpoort, J. Chem. Phys. 106, 3287 (1997).

${ }^{15}$ C. de Graaf, R. Broer, and W. C. Nieuwpoort, Chem. Phys. Lett. 271, 372 (1997).

${ }^{16}$ G. J. M. Janssen and W. C. Nieuwpoort, Phys. Rev. B 38, 3449 (1988).

${ }^{17}$ K. Terakura, T. Oguchi, A. R. Williams, and J. Klüber, Phys. Rev. B 30, 4734 (1984).

${ }^{18}$ Z.-X. Shen, R. S. List, D. S. Dessau, B. O. Wells, O. Jepsen, A. J. Arko, R. Barttlet, C. K. Shih, F. Parmigiani, J. C. Huang, and P. A. P. Lindberg, Phys. Rev. B 44, 3604 (1991).

${ }^{19}$ T. C. Leung, C. T. Chan, and B. N. Harmon, Phys. Rev. B 44, 2923 (1991).

${ }^{20} \mathrm{Ph}$. Dufek, P. Blaha, V. Sliwko, and K. Schwarz, Phys. Rev. B 49, 10170 (1994).

${ }^{21}$ T. Bredow and A. R. Gerson, Phys. Rev. B 61, 5194 (2000).

${ }^{22}$ Z. Szotek, W. M. Temmerman, and H. Winter, Phys. Rev. B 47, 4029 (1993).
${ }^{23}$ S. L. Dudarev, G. A. Botton, S. Y. Savrasov, Z. Szotek, W. M. Temmerman, and A. P. Sutton, Phys. Status Solidi A 166, 429 (1998).

${ }^{24}$ V. I. Anisomov, J. Zaanen, and O. K. Andersen, Phys. Rev. B 44, 943 (1991).

${ }^{25}$ V. I. Anisimov, I. V. Solovyev, M. A. Korotin, M. T. Czyzyk, and G. A. Sawatzky, Phys. Rev. B 48, 16929 (1993).

${ }^{26}$ S. Massidda, A. Continenza, M. Posternal, and A. Baldereschi, Phys. Rev. B 55, 13494 (1997).

${ }^{27}$ F. Aryasetiawan and O. Gunnarsson, Phys. Rev. Lett. 74, 3221 (1995).

${ }^{28}$ A. D. Becke, J. Chem. Phys. 98, 1372 (1993).

${ }^{29}$ A. D. Becke, Phys. Rev. A 38, 3098 (1988).

${ }^{30}$ A. D. Becke, J. Chem. Phys. 98, 5648 (1993).

${ }^{31}$ C. Lee, W. Yang, and R. G. Parr, Phys. Rev. B 37, 785 (1988).

${ }^{32}$ R. Colle and O. Salvetti, Theor. Chim. Acta 37, 329 (1975); 53, 55 (1979).

${ }^{33}$ R. Colle and O. Salvetti, J. Chem. Phys. 79, 1404 (1993).

${ }^{34}$ A. Ricca and C. W. Bauschlicher, J. Phys. Chem. 98, 12899 (1994).

${ }^{35}$ T. V. Russo, R. L. Martin, and P. J. Hay, J. Chem. Phys. 102, 8023 (1995).

${ }^{36}$ P. E. M. Siegbahn and R. H. Crabtree, J. Am. Chem. Soc. 119, 3103 (1997).

${ }^{37}$ C. Adamo and V. Barone, Chem. Phys. Lett. 274, 242 (1997).

${ }^{38}$ J. D. Talman and W. F. Shadwick, Phys. Rev. A 14, 36 (1976).

${ }^{39}$ J. B. Krieger, Y. Li, and G. J. Iafrate, Phys. Rev. A 45, 101 (1992).

${ }^{40}$ A. Gorling and M. Levy, Phys. Rev. A 50, 196 (1994).

${ }^{41}$ A. Gorling and M. Levy, Int. J. Quantum Chem., Symp. 29, 93 (1995).

${ }^{42}$ M. Stadele, M. Moukara, J. A. Majewski, P. Vogl, and A. Gorling, Phys. Rev. B 59, 10031 (1999).

${ }^{43}$ S. Ivanov, S. Hirata, and R. J. Bartlett, Phys. Rev. Lett. 83, 5455 (1999).

${ }^{44}$ T. Grabo, T. Kreibich, S. Kurth, and E. K. U. Gross, in Strong Coulomb Correlations in Electronic Structure: Beyond the Local Density Approximation, edited by V. I. Anisimov (Gordon and Breach, Tokyo, 1998), and references therein.

${ }^{45}$ E. Clementi, J. Chem. Phys. 93, 2591 (1990). 
${ }^{46}$ P. M. W. Gill, B. G. Johnson, J. A. Pople, and M. J. Frisch, Int. J. Quantum Chem. 26, 319 (1992).

${ }^{47}$ F. Illas, J. Rubio, J. M. Ricart, and G. Pacchioni, J. Chem. Phys. 105, 7192 (1996).

${ }^{48}$ V. R. Saunders, R. Dovesi, C. Roetti, M. Causà, N. M. Harrison, R. Orlando, and C. M. Zicovich-Wilson, computer code CRYSTAL, University of Torino, Torino, 1998.

${ }^{49}$ A. Wander, B. Searle, and N. M. Harrison, Surf. Sci. 458, 25 (2000)

${ }^{50}$ J. Muscat, A. Wander, and N. M. Harrison, Chem. Phys. Lett. 342, 397 (2001).

${ }^{51}$ J. K. Perry, J. Tahir-Kheli, and W. A. Goddard, Phys. Rev. B 63, 144510 (2001).

${ }^{52}$ R. L. Martin and F. Illas, Phys. Rev. Lett. 79, 1539 (1997).

${ }^{53}$ F. Illas and R. L. Martin, J. Chem. Phys. 108, 2519 (1998).

${ }^{54}$ P. D. V. DuPleiss, S. J. Van Tonder, and L. Alberts, J. Phys. C 4, 1983 (1971).

${ }^{55}$ B. E. F. Fender, A. J. Jacobson, and F. A. Wedgewood, J. Chem. Phys. 48, 990 (1968).

${ }^{56}$ A. K. Cheetham and D. A. O. Hope, Phys. Rev. B 27, 6964 (1983).

${ }^{57}$ M. T. Hutchings and E. J. Samuelsen, Phys. Rev. B 6, 3447 (1972).

${ }^{58}$ R. Shanker and R. A. Singh, Phys. Rev. B 7, 5000 (1973).

${ }^{59}$ D. Muñoz, I. de P. R. Moreira, and F. Illas, Phys. Rev. Lett. 84, 1579 (2000).

${ }^{60}$ I. de P. R. Moreira, F. Illas, C. J. Calzado, J. F. Sanz, J. P. Malrieu, N. Ben Amor, and D. Maynau, Phys. Rev. B 59, R6593 (1999).

${ }^{61}$ C. de Graaf, I. de P. R. Moreira, F. Illas, and R. L. Martin, Phys. Rev. B 60, 3457 (1999).

${ }^{62}$ C. de Graaf and F. Illas, Phys. Rev. B 63, 014404 (2000).

${ }^{63}$ H. A. Alperin, Phys. Rev. Lett. 6, 55 (1961).

${ }^{64}$ H. A. Alperin, J. Phys. Soc. Jpn., Suppl. B 17, 12 (1962).

${ }^{65}$ H. Chang, J. F. Harrison, T. A. Kaplan, and S. Mahanti, Phys. Rev. B 49, 15753 (1994).

${ }^{66}$ Lecture Notes in Chemistry, edited by C. Pisani, R. Dovesi, and C. Roetti (Springer, Heidelberg, 1988), Vol. 48.

${ }^{67}$ Lecture Notes in Chemistry, edited by C. Pisani (SpringerVerlag, Heidelberg, 1996), Vol. 67.

${ }^{68}$ M. D. Towler, N. L. Allan, N. M. Harrison, V. R. Saunders, W. C. Mackrodt, and E. Aprà, Phys. Rev. B 50, 5041 (1994).

${ }^{69}$ J. M. Ricart, R. Dovesi, C. Roetti, and V. R. Saunders, Phys. Rev. B 52, 2381 (1995); see also erratum in ibid. 55, 15942 (1997).

${ }^{70}$ P. Reinhardt, I. de P. R. Moreira, C. de Graaf, F. Illas, and R. Dovesi, Chem. Phys. Lett. 319, 625 (2000).

${ }^{71}$ R. Dovesi, C. Roetti, C. Freyria-Fava, M. Prencipe, and V. R. Saunders, Chem. Phys. 156, 11 (1991).

${ }^{72}$ M. Catti, R. Dovesi, A. Pavese, and V. R. Saunders, J. Phys.: Condens. Matter 3, 4151 (1991).

${ }^{73}$ R. L. Martin, J. Chem. Phys. 98, 8691 (1993).

${ }^{74}$ F. Illas, J. Casanovas, M. A. García-Bach, R. Caballol, and O. Castell, Phys. Rev. Lett. 71, 3549 (1993).

${ }^{75}$ J. Casanovas and F. Illas, Phys. Rev. B 50, 3789 (1994).

${ }^{76}$ J. Casanovas and F. Illas, J. Chem. Phys. 101, 7683 (1994).

${ }^{77}$ J. Casanovas, J. Rubio, and F. Illas, Phys. Rev. B 53, 945 (1996).

${ }^{78}$ I. de P. R. Moreira and F. Illas, Phys. Rev. B 55, 4129 (1997).

${ }^{79}$ P. Reinhardt, M. P. Habas, R. Dovesi, I. de P. R. Moreira, and F.
Illas, Phys. Rev. B 59, 1016 (1999).

${ }^{80}$ I. de P. R. Moreira and F. Illas, Phys. Rev. B 60, 5179 (1999).

${ }^{81}$ N. W. Winter and R. M. Pitzer, J. Chem. Phys. 89, 446 (1988).

${ }^{82}$ C. Sousa, J. Casanovas, J. Rubio, and F. Illas, J. Comput. Chem. 14, 680 (1993).

${ }^{83}$ G. Pacchioni, A. M. Ferrari, A. M. Marquez, and F. Illas, J. Comput. Chem. 18, 617 (1996).

${ }^{84}$ R. Caballol, O. Castell, F. Illas, J. P. Malrieu, and I. de P. R. Moreira, J. Phys. Chem. 101, 7860 (1997).

${ }^{85}$ F. Illas, N. López, I. de P. R. Moreira, and M. García-Hernández, in Metal-ligand Interactions in Chemistry, Physics, and Biology, Vol. 546 of NATO Advanced Study Institute, Series C: Mathematical and Physical Sciences, edited by N. Russo and D. R. Salahub (Kluwer Academic, Dordrecht, 2000), p. 129.

${ }^{86}$ F. Illas, I. de P. R. Moreira, C. de Graaf, and V. Barone, Theor. Chem. Acc. 104, 265 (2000).

${ }^{87}$ B. O. Ross, Advances in Chemical Physics: Ab Initio Methods in Quantum Chemistry II (Wiley, Chichester, England, 1987), Chap. 69, p. 399.

${ }^{88}$ K. Andersson, P.-A. Malmqvist, B. O. Roos, A. J. Sadlej, and K. Wolinski, J. Phys. Chem. 94, 5483 (1990).

${ }^{89}$ K. Andersson, P.-A. Malmqvist, and B. O. Roos, J. Chem. Phys. 96, 1218 (1992).

${ }^{90}$ J. Miralles, O. Castell, R. Caballol, and J. P. Malrieu, Chem. Phys. 172, 33 (1993).

${ }^{91}$ M. J. Frisch et al., computer code GAUSSIAN 98, revision A.6, Gaussian Inc., Pittsburgh PA, 1998.

${ }^{92}$ K. Andersson, M. R. A. Blomberg, M. P. Fülscher, G. Karlström, R. Lindh, P.-A. Malmqvist, P. Neogrády, J. Olsen, B. O. Roos, A. J. Sadlej, M. Schütz, L. Seijo, L. Serrano-Andrés, P. E. M. Siegbahn, and P.-O. Widmark, computer code MOLCAS version 4, University of Lund, Sweden, 1997.

${ }^{93}$ D. Maynau and N. Ben Amor, CASDI suite of programs, Toulouse, 1997

${ }^{94}$ N. Ben Amor and D. Maynau, Chem. Phys. Lett. 286, 211 (1998).

${ }^{95}$ J. P. Daudey, adaptation of the MOTRA module to the HONDOCIPSI-CASDI package (unpublished).

${ }^{96}$ P.-O. Widmark, P.-A. Malmqvist, and B. O. Roos, Theor. Chim. Acta 77, 291 (1990).

${ }^{97}$ K. Pierloot, B. Dumez, P.-O. Widmark, and B. O. Roos, Theor. Chim. Acta 90, 87 (1995).

${ }^{98}$ R. Pou-Amérigo, M. Merchán, I. Nebot-Gil, P.-O. Widmark, and B. O. Roos, Theor. Chim. Acta 92, 149 (1995).

${ }^{99}$ S. H. Vosko, L. Wilk, and M. Nusair, Can. J. Phys. 58, 1200 (1980).

${ }^{100}$ J. P. Perdew, K. Burke, and Y. Wang, Phys. Rev. B 54, 16533 (1996), and references therein.

${ }^{101}$ J. P. Perdew, J. A. Chevary, S. H. Vosko, K. A. Jackson, M. R. Pederson, D. J. Singh, and C. Fiolhais, Phys. Rev. B 46, 6671 (1992).

${ }^{102}$ N. Uchida and A. Saito, J. Acoust. Soc. Am. 51, 1602 (1972).

${ }^{103}$ J. Wang, E. S. Fisher, and M. H. Manghnzimi, Chin. Phys. Lett. 8, 153 (1991).

${ }^{104}$ P. S. Bagus, F. Illas, C. Sousa, and G. Pacchioni, Electronic Properties of Solids Using Cluster Methods, edited by T. A. Kaplan and S. D. Mahanti (Plenum, New York, 1995), p. 93.

${ }^{105}$ H. Chevreau, I. de P. R. Moreira, B. Silvi, and F. Illas, J. Phys. Chem. A 105, 3570 (2001). 
${ }^{106}$ J. C. Slater and J. H. Wood, Int. J. Quantum Chem., Symp. 4, 3 (1971).

${ }^{107}$ J. F. Janak, Phys. Rev. B 18, 7165 (1978).

${ }^{108}$ J. P. Perdew, R. G. Parr, M. Levy, and J. L. Balduz, Phys. Rev. Lett. 49, 1691 (1982).

${ }^{109}$ A. Görling, Phys. Rev. A 54, 3912 (1996).

${ }^{110}$ A. Savin, C. J. Umrigar, and X. Gonze, Chem. Phys. Lett. 288, 391 (1998).

${ }^{111}$ E. J. Baerends and O. V. Gritsenko, J. Phys. Chem. A 101, 5383 (1997).

${ }^{112}$ F. Illas, I. de P. R. Moreira, C. de Graaf, O. Castell, and J. Casanovas, Phys. Rev. B 56, 5069 (1997).

${ }^{113}$ The primitive 2-atom crystalline cell is related to the conventional 8-atom crystalline cell by an $A$ $=(-1,1,1,1,-1,1,1,1,-1)$ expansion matrix of the direct vectors and the 64-atom AF2 conventional cell is constructed by doubling the conventional 8-atom crystallographic cell in each direction of the space [i.e., the $B=(2,0,0,0,2,0,0,0,2)$ expansion matrix]. On the other hand, the 4-atom primitive AF2 cell is constructed by a $C=(0,1,1,1,0,1,1,1,0)$ expansion matrix of the 2 -atom primitive cell. Hence, by a simple matrix operation the $h, k, l$ set of indices referred to the conventional magnetic cell are related to a set $h_{m}, k_{m}, l_{m}$ of indices referred to the 4-atom primitive $\mathrm{AF} 2$ cell by the transformation $C B^{-1} A^{-1}$ $=(1 / 2,1 / 4,1 / 4,1 / 4,1 / 2,1 / 4,1 / 4,1 / 4,1 / 2)$. 